\title{
Study On The Influence Of FC Evaporator Structure On The Internal Flow Field
}

\author{
Chong Zhao, a, Ruozheng Li ${ }^{1, b}$, Peilin Zhang1, c, Jilin Teng ${ }^{1, d}$ \\ Ying Tian ${ }^{1,}$, Wenming $\mathrm{Ma}^{1, \mathrm{f}}$ \\ ${ }^{1}$ Beijing guodian futong science and technology development Co.Ltd, Beijing, China, 100070 \\ ayeah231@163.com, 'Irzheng0929@sian.com, 'czhangpeilin@sgepri.sgcc.com.cn, dhdtjl@163.com \\ ettsherry@126.com, ${ }^{f}$ mwmwenming@126.com
}

Keywords: FC (Forced circulation) evaporator;Internal flow field;Finite element analysis; Multiphase flow

Abstract. Take the structure of the FC evaporator for desulfurization wastewater from power plant as the research object, establish model of the finite element analysis, and the internal flow simulation is carried out. Compare the characteristics of the flow field in the evaporator with the different structure parameters, and study the particle size distribution. The results show that the angle of the inlet tube and the angle of the discharge outlet can affect the local flow field in the evaporator, thus affecting the crystallization process. By adjusting the above Angle and optimizing the internal flow field, the particle phase can be fully mixed to avoid the solid phase segregation.

\section{Introduction}

As the core equipment of salt industry, the evaporative crystallizer is more and more applied to the evaporative crystallization process of high-salt wastewater with the proposal of zero discharge of waste water. Positive cyclic axial feed evaporator is the wide application of a kind of shape, compared with the traditional tangential feeding evaporator, it can avoid the hot short circuit and the vortex loss problem, and reduce the loss of temperature difference ${ }^{[1]}$. But in the structure design of the axial feed evaporator, it is also based on the empirical value. This causes the evaporator to have a floating dead zone, which affects the crystallization reaction, and causes the inner wall to scale.

In this paper, using the Fluent software to simulate the gas-liquid two phase and gas-liquid-solid three-phase flow respectively, based on this, research the influence of tanks and circular tube structure parameters on the internal flow field ${ }^{[2]}$. At last, through analysis and comparison between the results on the structural parameters of evaporator, summarize the key points for the equipment design, which can provide reasonable scientific basis to improve the performance of the evaporator.

\section{Simulation method}

Firstly calculate the free surface formed by the liquid flowing out of the bellmouth, which provides the basis for structural design, and then add the discrete particles into the flow field, study the movement rule of solid-liquid mixed phase ${ }^{[3,4]}$.

\section{Structure of the evaporator}

Based on the analysis results of FGD wastewater ${ }^{[5]}$, one FC evaporator of $4 \mathrm{t} / \mathrm{h}$ evaporation capacity is designed. The initial structure parameters are shown in table 1. 
Table 1. initial structure parameters

\begin{tabular}{ccc}
\hline item & unit & value \\
\hline diameter & $\mathrm{mm}$ & 2135 \\
cone angle & $\circ$ & 35 \\
straight height & $\mathrm{mm}$ & 5000 \\
circulating pipe diameter & $\mathrm{mm}$ & 720 \\
bellmouth angle & $\circ$ & 13.5 \\
circulating pipe outlet angle & $\circ$ & 90 \\
\hline
\end{tabular}

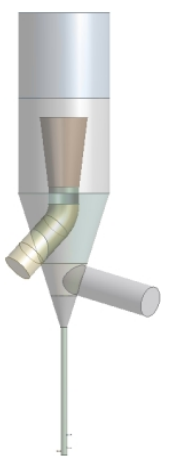

Fig.1. 3D model of FC evaporator

\section{Grid division}

The 3D model in fig. 1 is divided into grids, the grid type is hybrid grid, and this type can not only reduce the number of meshes, but also accelerate the convergence. Grid sections are shown in fig. 2 .

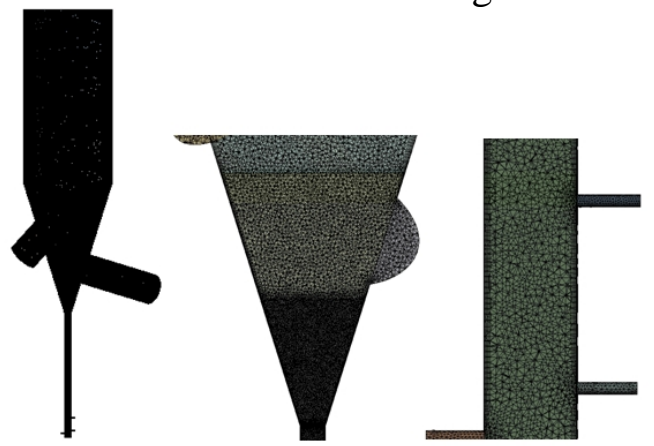

Fig.2. Finite element model of the FC evaporator

\section{Simulation model}

In this paper, air is regarded as incompressible flow. For incompressible Newtonian fluids, the continuity equation and the momentum equation can be expressed as follows:

$$
\begin{aligned}
& \nabla \cdot \dot{u}=0 \\
& \frac{\partial(\rho \dot{u})}{\partial t}+\nabla \cdot(\rho u u)=-\nabla p+\left[\mu\left(\nabla \stackrel{r}{r}+\left(\nabla u^{r}\right)^{T}\right)\right]+F_{s}+\rho \stackrel{r}{g}
\end{aligned}
$$

In the VOF model, the interface between phase and phase is completed by solving the continuity equation of a phase or multiphase volume fraction. For phase $n$, the equation is as follows:

$$
\frac{\partial F_{n}}{\partial t}+\stackrel{r}{u_{n}} \cdot \nabla F_{n}=0
$$

Formula (3) is used to solve the volume fraction of the second phase (the gas phase), and the calculation of the main phase volume fraction is based on the following constraints:

$$
\sum_{n=1}^{2} F_{n}=1
$$


The surface tension in equation (2) is processed by a continuous surface force model, in which surface tension is along the constant surface and considered that the tension only perpendicular to the interface. Add this force to the momentum square source term, and the form is as follows:

$$
\begin{aligned}
& F_{s}=\sigma \frac{\rho \kappa \nabla F_{1}}{0.5\left(\rho_{g}+\rho_{l}\right)} \\
& \kappa=\nabla \cdot \hat{n}, \hat{n}=\frac{n}{|n|}, n=\nabla F_{n}
\end{aligned}
$$

The trajectory of discrete phase particles is forecasted by calculating the force of the particles (not including the intergranular interaction), and tracking particle trajectory takes Lagrangian coordinates as the reference frame. The force equation involves the inertial force and other forces, which can be written in cartesian coordinates as:

$$
\frac{d u_{p}}{d t}=F_{D}\left(u-u_{p}\right)+\frac{g\left(\rho_{p}-\rho\right)}{\rho_{p}}+F
$$

Where $\mathrm{F}$ is the additional accelerator, $\mathrm{F}_{\mathrm{D}}\left(\mathrm{u}-\mathrm{u}_{\mathrm{p}}\right)$ is the drag force of the unit particle mass.

$$
F_{D}=\frac{18 \mu}{\rho_{p} d_{p}^{2}} \cdot \frac{C_{p} \mathrm{Re}}{24}
$$

Where $u$ is the liquid phase velocity, $u_{p}$ is the particle velocity, $\mu$ is the fluid viscosity, $\rho$ is the fluid density, $\rho_{p}$ is the particle density, $d_{p}$ is the particle diameter. Re is the relative Reynolds number, which is defined as:

$$
\operatorname{Re}=\frac{\rho d_{p}\left|u_{p}-u\right|}{\mu}
$$

The drag coefficient $\mathrm{C}_{\mathrm{D}}$ can be obtained from the following formula:

$$
C_{D}=a_{1}+\frac{a_{2}}{\operatorname{Re}}+\frac{a_{3}}{\operatorname{Re}^{2}}
$$

$\mathrm{a}_{1}, \mathrm{a}_{2}$ and $\mathrm{a}_{3}$ are constants, with have different values in different Re ranges ${ }^{[6]}$.

\section{Boundary conditions}

The fluid in the evaporator contains only saturated salt solutions and salt granule, and the material properties are shown in table 2.

Table 2. material properties item

\begin{tabular}{ccc}
\hline item & density $\left(\mathbf{k g} / \mathbf{m}^{\mathbf{3}}\right)$ & viscosity $\left(\mathbf{k g} / \mathbf{m}^{\mathrm{s}}\right)$ \\
\hline solutions & 1200 & 0.001003 \\
granule & 2100 & 0.2 \\
\hline
\end{tabular}

The operating conditions of liquid flow in evaporator are shown in table 3.

Table 3. operation paramete

\begin{tabular}{ccc}
\hline parameter & unit & \\
\hline influent flow of the evaporator & $\mathrm{m}^{3} / \mathrm{h}$ & 2600 \\
inflow 1 of the salt leg & $\mathrm{m}^{3} / \mathrm{h}$ & 0.7 \\
inflow 2 of the salt leg & $\mathrm{m}^{3} / \mathrm{h}$ & 0.3 \\
outflow of the salt leg & $\mathrm{m}^{3} / \mathrm{h}$ & 4.5 \\
\hline
\end{tabular}

\section{Analysis of simulation result}

\section{Influence of inlet bellmouth angle to the flow field}

The feeding bellmouth has the function of evenly distributing liquid, and can also relieve the impact of liquid. Set up three bellmouth angle of $9^{\circ} 、 13.5^{\circ}$ and $18^{\circ}$, and compare the flow field near the surface. The inflow and outflow are based on the data in table 3. 


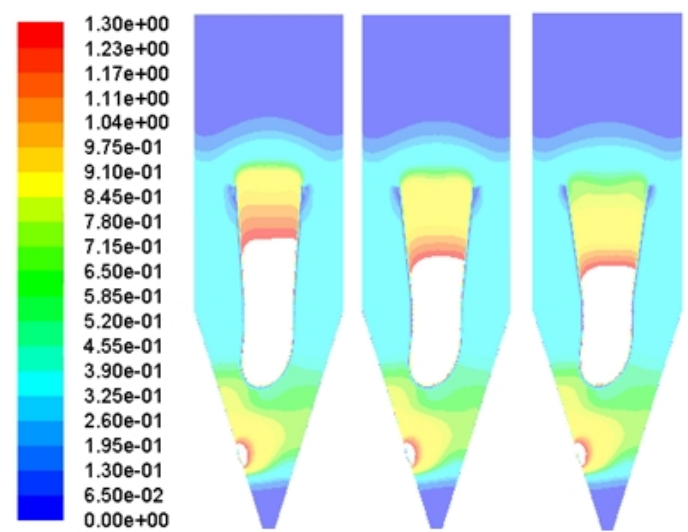

Fig.3. velocity distribution of the liquid phase

(From left to right bellmouth opening angle of $9^{\circ}$ and $13.5^{\circ}$ and $18^{\circ}$ )

Fig. 3 shows the velocity distribution of the liquid phase. From the figure, the larger the angle of the bellmouth, the smaller the flow velocity of the liquid phase in the loop tube is and the more gentle the liquid surface is. At the same time, there are some dark blue low-speed areas on the outer edge of the bellmouth, and the flow of liquid in the low-speed areas is close to stagnation, which is bad for crystallization and easy to scale. The velocity vector graph and local amplification are used for further analysis, which shows as Fig.4. It can be seen from the figure that a flow vortex at the edge of the bellmouth forms. It is obvious that the larger the Angle of the opening angle, the smaller the vortex is, the smaller the area of the dead zone is. This allows the liquid to more fully participate in the cycle and crystallization reaction.

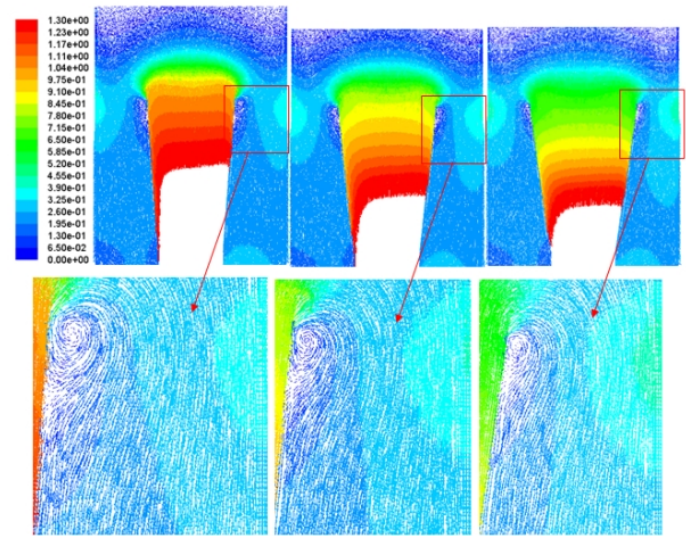

Fig.4. Velocity vector graph and local amplification

(From left to right bellmouth opening angle of $9^{\circ}$ and $13.5^{\circ}$ and $18^{\circ}$ )

\section{Influence of the outlet angle to the flow field}

Considering from equipment manufacturing, setting the outlet as $90^{\circ}$, with the circular tube perpendicular to cone bucket, it is more convenient for processing. This article will replace pipe connecting elbow with special-shaped, angle can be adjusted to $63^{\circ}$. So keep the bellmouth opening angle as $18^{\circ}$, set up two kinds of outlet angle as $90^{\circ}$ and $63^{\circ}$, carry on simulation comparison and analysis.

As shown in Fig.5 and Fig.6 is the local magnification of streamline in the circulating outlet,it can be seen when the circulating outlet angle at $90^{\circ}$, the streamline at the joint of evaporator and circulating outlet suddenly swerves, the upper in the tube has a low pressure area, and the flow stagnates, which is bad for circulation. Angle decreases to $63^{\circ}$, the liquid flow becomes more smooth, and low pressure area decrease obviously. This reduces flow stagnation and avoids excessive temperature loss. 


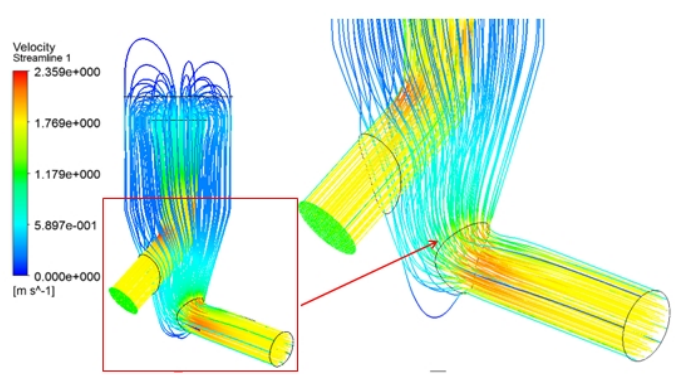

Fig.5. local magnification of streamline with the angle at $90^{\circ}$

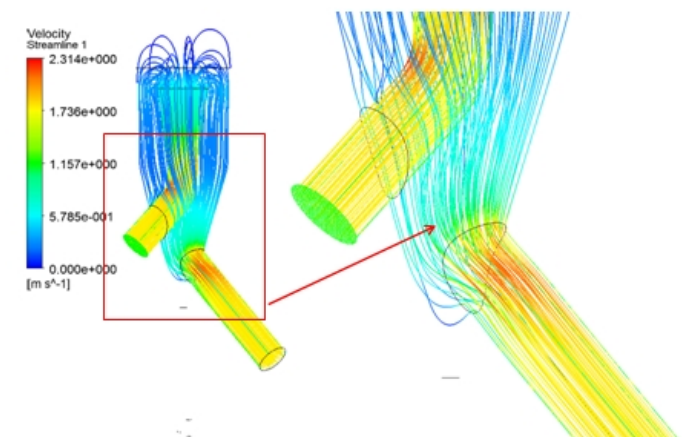

Fig.6. local magnification of streamline with the angle at $63^{\circ}$

\section{Discrete phase calculation results}

After the calculation of the fluid is completed, the discrete phase particles are added to simulate the movement of the crystal salt inside. At the beginning of the calculation, the particles were continuously added to the circulation feeding nozzle until the stable output of particles begins. And the distribution of the grain concentration in the evaporator is investigated. The particle attributes are shown in table 4.

Table 4. particle attributes

\begin{tabular}{ccc}
\hline parameter & unit & \\
\hline Density & $\mathrm{kg} / \mathrm{m} 3$ & 2100 \\
minimum size & $\mathrm{mm}$ & 0.2 \\
maximum size & $\mathrm{mm}$ & 0.8 \\
average Size & $\mathrm{mm}$ & 0.4 \\
mass flux & $\mathrm{kg} / \mathrm{s}$ & 151.67 \\
\hline
\end{tabular}

The boundary properties of discrete phases in different locations are shown in table 5.

Table 5. boundary properties of discrete phases

\begin{tabular}{cc}
\hline location & boundary properties \\
\hline circulating inlet & escape \\
circulating outlet & escape \\
Inlet 1 of salt leg & reflect \\
Inlet 2 of salt leg & reflect \\
\hline
\end{tabular}

As shown in Fig.7 is particle concentration distribution at different time. The left is showing the particle concentration distribution at the 18th second, there is high concentration of particles near the bellmouth, at this time the evaporator is not completely filled with particles. The right is showing the particle concentration distribution at the 43th second, and the particles are completely filled with evaporator. It can be seen from the figure that the concentration distribution in the evaporator tends to be uniform as time increases. The final average concentration is about $225 \mathrm{~kg} / \mathrm{m}^{3}$, which is very similar to the fixed solid-liquid ratio, indicating that the added particles can be fully mixed with the liquid. In addition, the mixture of solid and liquid can provide a good crystal environment, which can eliminate the saturation in time. The final product has a uniform salinity, and it can effectively prevent scale on inner wall ${ }^{[7]}$. 


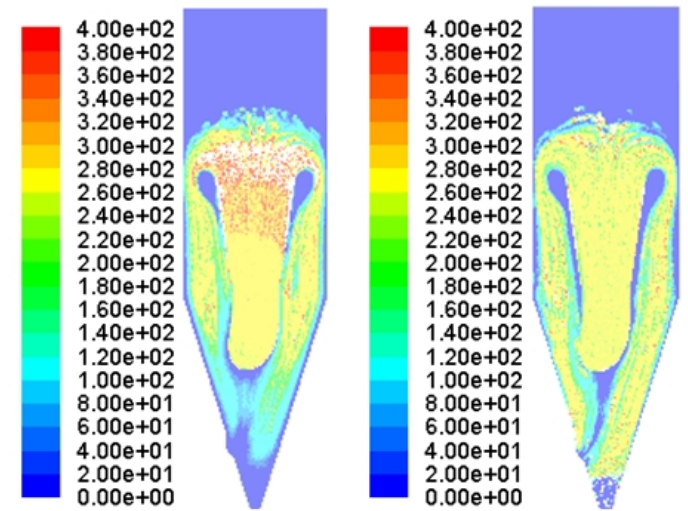

Fig.7. particle concentration distribution(18s and $43 \mathrm{~s})$

Based on the discrete particle model of 1.3.2, the particle size distribution of product salt (discharge from salt leg) is investigated and compared with those return to evaporator (to the circulation outlet). Table 6 shows the size distribution data of the particle from circulation outlet and the discharge outlet. The average size of the particle from circulating outlet is $0.396 \mathrm{~mm}$, and the average size of the particle discharge of salt legs is $0.729 \mathrm{~mm}$. It can be seen that under this design, it can get the larger product's size, and big particle size is very beneficial to the purity of the product.

Table 6. size distribution data of the particle

\begin{tabular}{ccc}
\hline particle size $(\mathbf{m m})$ & size distribution from circulating outlet & size distribution from discharge outlet \\
\hline 0.200 & 33.23 & 0 \\
0.333 & 21.90 & 0 \\
0.467 & 16.50 & 1.728 \\
0.533 & 11.98 & 4.675 \\
0.667 & 9.240 & 44.89 \\
0.800 & 7.156 & 48.70 \\
\hline
\end{tabular}

Fig. 8 shows the bar chart of particle content with different diameter, it can be more intuitive to see from the chart that content of small particles size from the circulation outlet is more, content of big particles size from the discharge outlet is more, which meet the requirements of the production of large grain of salt.

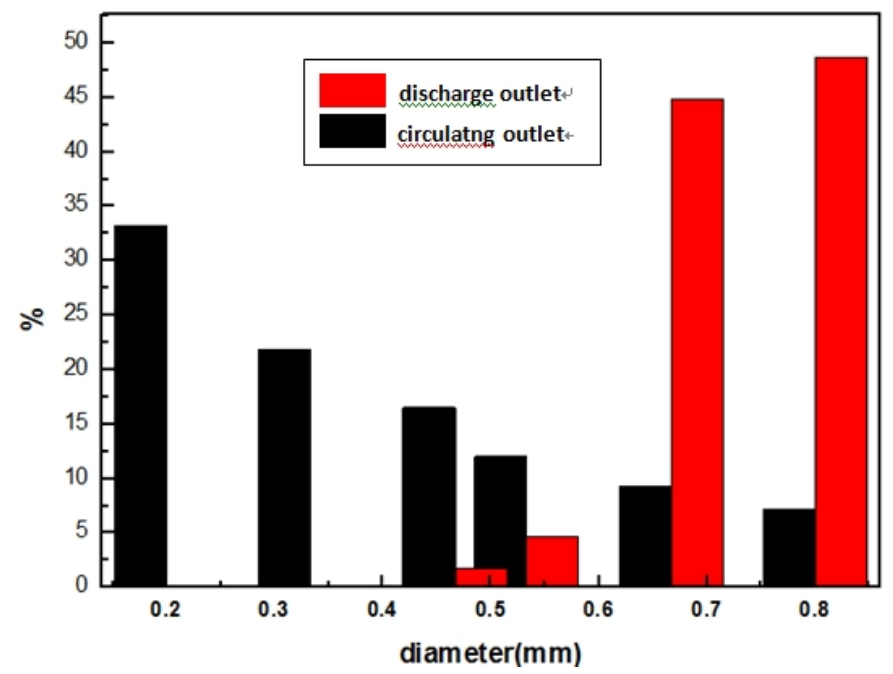

Fig.8. particle content with different size from circulation outlet and discharge outlet

\section{Conclusion}

The flow tends to form a vortex at the outer edge of feeding bellmouth outlet, which affects the overall flow field. Increasing the angle of the bellmouth in a certain range can significantly reduce the area of this vortex and enhance the liquid circulation. 
Making the circulating outlet parallel with the evaporator can promote the flow of the circulating fluid more gentle, reduce the flow stagnation and avoid excessive temperature loss.

After optimizing the Angle of inlet bellmouth and circulating outlet, under the circulation flow rate of $2600 \mathrm{~m} 3 / \mathrm{h}$, more than $90 \%$ particle that above $0.66 \mathrm{~mm}$ can be discharged, and more than $70 \%$ particle that below $0.5 \mathrm{~mm}$ stays in evaporator. Product can keep large particle size. This not only guarantees the requirements of particle size, but also improves the purification of the product.

\section{Acknowledgements}

This work was financially supported by the Innovation Program of NARI Group Corporation (FTZX 201701).

\section{References}

[1] Luo Dazhong: China Well And Rock Salt, Vol. 34(2003), p. 9-14.

[2] Qi Chunhua, Fan Mingming, Xie Feng, Lv Qingchun, Ruan Guoling, Zhao Jun: China Water \& Wastewater, Vol. 25 (2009), p. 87-91.

[3] Yonggui Xu: Tianjin, Tianjin University, 2013, p. 129-133.

[4] Xia Mi, Li Yi, Li Fengqin: Journal of Mechanical \& Electrical Engineering, Vol. 32(2015), p. $1555-1563$.

[5] Shi Hu, Shaofeng Ding, Zhaoshi Fan: Clean Coal Technology, Vol. 21 (2015), p. 129-133.

[6] SA Morsi, AJ Alexander: Journal of Fluid Mechanics, Vol. 55 (1972), p. 193-208.

[7] Wei Zongsheng: Chenmical Engineering Design, Vol. 10 (2000), p. 30-34. 